Southern Illinois University Carbondale

OpenSIUC

Articles

Department of Electrical and Computer

Engineering

Fall 11-23-2016

\title{
Low Rank and Sparsity Analysis Applied to Speech Enhancement via Online Estimated Dictionary
}

Pengfei Sun

Southern Illinois University Carbondale

Jun Qin

Southern Illinois University Carbondale, jqin@siu.edu

Follow this and additional works at: http://opensiuc.lib.siu.edu/ece_articles

(c) 2015 IEEE. Personal use of this material is permitted. Permission from IEEE must be obtained for all other users, including reprinting/ republishing this material for advertising or promotional purposes, creating new collective works for resale or redistribution to servers or lists, or reuse of any copyrighted components of this work in other works.

\section{Recommended Citation}

Sun, Pengfei and Qin, Jun. "Low Rank and Sparsity Analysis Applied to Speech Enhancement via Online Estimated Dictionary." IEEE Signal Processing Letters 23, No. 12 (Fall 2016): 1862-1866. doi:10.1109/LSP.2016.2627029.

This Article is brought to you for free and open access by the Department of Electrical and Computer Engineering at OpenSIUC. It has been accepted for inclusion in Articles by an authorized administrator of OpenSIUC. For more information, please contact opensiuc@lib.siu.edu. 


\title{
Low Rank and Sparsity Analysis Applied to Speech Enhancement via Online Estimated Dictionary
}

\author{
Pengfei Sun, Student Member, and Jun Qin, Member, IEEE
}

\begin{abstract}
In this letter, we propose an online estimated dictionary based single-channel speech enhancement algorithm, which focuses on low-rank and sparse matrix decomposition. In the proposed algorithm, a noisy speech spectrogram can be decomposed into low rank background noise components and an activation of the online speech dictionary, on which both low-rank and sparsity constraints are imposed. This decomposition takes the advantage of local estimated exemplar's high expressiveness on speech components and also accommodates nonstationary background noise. The local dictionary can be obtained through estimating the speech presence probability (SPP) by applying Expectation-Maximal algorithm, in which a generalized Gamma prior for speech magnitude spectrum is used. The proposed algorithm is evaluated using signal-to-distortion ratio (SDR), and perceptual evaluation of speech quality (PESQ). The results show that the proposed algorithm achieves significant improvements at various SNRs when compared to four other speech enhancement algorithms, including improved Karhunen-Loeve transform (KLT) approach, SPP based MMSE (MMSE-SPP), NMF based RPCA (NMF-RPCA), and RPCA.
\end{abstract}

Index Terms-speech enhancement, online speech dictionary, low rank, sparsity, speech presence probability.

\section{INTRODUCTION}

$\mathbf{S}$ INGLE channel speech enhancement is a key issue in speech processing, aiming to improve the performance of speech communication systems in noisy environments. Recently-developed robust principal component analysis (RPCA) has been shown effective in separating the speech components from background noise [4], [14], [16]. This approach decomposes the spectrogram matrix as the sum of a sparse matrix and a low-rank matrix, representing speech and noise, respectively [15]. Based on the observation that unpredictable background noise is often less spectrally diverse than the foreground speech, it regards that sparse matrix represents the speech components while noise is included in the low rank matrix [1]. Because the activation of the lowrank components can be temporally variable, this unsupervised decomposition can accommodate nonstationary noise [13]. However, the targeted speech may also contain low rank components described by limited spectral bases, and these low rank speech components can be wrongly decomposed into noise matrix. Thus unsupervised decomposition may not provide accurate separation of foreground and background in certain circumstance, e.g., nonstationary noise [11].

P.F. Sun and J. Qin, are with the Department of Electrical and Computer Engineering, Southern Illinois University, Carbondale, IL, 62901 USA (email: sunpengfei@siu.edu; jqin@siu.edu).

Copyright (c) 2015 IEEE. Personal use of this material is permitted. However, permission to use this material for any other purposes must be obtained from the IEEE by sending a request to pubs- permissions@ieee.org.
To avoid simply using sparsity to characterize speech spectrogram, incorporating the knowledge about the likely form of the targeted speech has been introduced [2], [11]. Using nonnegative matrix factorization (NMF) technique, pre-learned explicit speech dictionary [3], [12] based on large dataset can improve the performance of RPCA. The advantage of the offline-trained global dictionary based RPCA approach is its ability to maintain the flexibility to distinguish speech and noise, and also avoid wrongly decomposing the low-rank speech components into noise subspace. However, offlinelearned global basis spectra may either lead to a non-'sparse' activation matrix or wrongly interpret background noise as speech. This is intrinsically due to overfitting caused by limited local speech eigenvectors and shared basis spectra between speech and noise [3]. Recent study [10] indicates that exemplar based dictionary can be more effective on covering speech spectra convex hull. In addition, online trained dictionary [3] presents a solution to achieve higher noise reduction whereas lower speech distortion. To exploit the advantages of both, a natural idea is to develop an online learned exemplar-structure dictionary.

In this study, we propose an online estimated dictionary based low rank and sparse decomposition (LDLSD) algorithm. The developed local dictionary estimation inherits the merit of both exemplar's efficient explanation on the speech manifold and adaptive speech subspace that alleviating the immediate speech distortion [4]. In LDLSD algorithm, a semi-supervised Expectation-Maximum (EM) algorithm based on a generalized Gamma speech distribution model is used to calculate the speech presence probability (SPP), and further obtain the local estimated exemplar. Moreover, the activation matrix of speech is optimized with respect to both low rank and sparsity constraints to suppress the noise entries mixed in the exemplar.

\section{Proposed Speech Enhancement Method}

\section{A. Model}

RPCA related approaches generally decompose an input spectrogram matrix $Y \in \mathbb{R}^{N \times M}$ as the summation of a lowrank matrix $L$ reflecting less spectrally diverse noise, and a sparse matrix $S$, representing the sparsity of speech energy as shown in (1). Outlying entries $E$ are frequently added to provide a relaxed estimation on the noise residuals [1], [14].

$$
\begin{array}{ll}
\min _{S, L} & \|S\|_{1}+\gamma_{l}\|L\|_{*}+\gamma_{e}\|E\|_{F}^{2} \\
\text { s.t. } & Y=S+L+E
\end{array}
$$

$\|\cdot\|_{*}$ is the nuclear norm of the matrix. The sparsity of $S$ is measured by the $L_{1}$ norm $\|\cdot\|_{1}$, and the Frobenious 
norm is used to represent the noise residual. Based on the observation that speech spectrogram is sparsity in short time period but with repeated structures (i.e., low-rank) within several consecutive segments, $S$ in (1) can be replaced by $Y_{1} S$, where as an explicit dictionary of speech spectral templates, $Y_{1}$ is multiplied by a set of temporal activation $S$. Generally, $Y_{1}$ is a global offline trained dictionary [2]. However, such kind of final learned dictionary may lose focus on the intermediate estimation of the local speech, and accordingly, is not necessarily good enough to explain current speech frame. Instead, an online speech exemplar can be estimated based on SPP given by

$$
Y_{1}=Y \odot \mathcal{P}
$$

$\odot$ refers to element-wise product of matrices, and $\mathcal{P}$ is the $\mathrm{SPP}$, for each frequency bin defined as

$$
\begin{aligned}
\mathcal{P}_{j} & =p\left\{z_{1} \mid y_{j}, \lambda\right\} \\
& =\frac{p\left(y_{j} \mid z_{1}, \lambda\right) p\left(z_{1}\right)}{\sum_{n=0}^{n=1} p\left(y_{j} \mid z_{n}, \lambda\right) p\left(z_{n}\right)}=\frac{p\left(y_{j} \mid z_{1}, \lambda\right) w_{1}}{\sum_{n=0}^{n=1} p\left(y_{j} \mid z_{n}, \lambda\right) w_{n}}
\end{aligned}
$$

where index $n \in\{1,0\}$ represents the case of speech present and absent, respectively. $w_{n}$ is the corresponding the a priori probability that $\left(\sum_{n} w_{n}=1\right) . \lambda \triangleq\left\{\mu_{n}, \sigma_{n}, w_{n}\right\}$ is the parameter set (i.e., mean, variance, and priori) for the statistic models of speech and noise. Considering noise obeys complex Gaussian distribution, $p\left(y_{j} \mid z_{0}, \lambda\right)$ should be Rayleigh distribution, and specifically, $p\left(y_{j} \mid z_{1}, \lambda\right)$ is assumed as generalized Gamma distribution given by [6]

$$
\begin{gathered}
p\left(y_{j} \mid z_{0}, \lambda\right)=\frac{y_{j}}{\delta^{2}} \exp \left\{-y_{j}^{2} / 2 \delta^{2}\right\} \\
p\left(y_{j} \mid z_{1}, \lambda\right)=\frac{\beta^{\nu}}{\Gamma(\nu)} y_{j}^{\nu-1} \exp \left\{-\beta y_{j}\right\}
\end{gathered}
$$

where

$$
\delta=\mu_{0} \sqrt{2 / \pi} ; \beta=\mu_{1} / \sigma_{1}^{2} ; \nu=\mu_{1}^{2} / \sigma_{1}^{2}
$$

Usually, the local exemplar $Y_{1}$ promises a sparse activation matrix $S$ as the representation of individual speech segment from the same subspace. However, in low SNR scenario, the estimated local exemplar will be highly corrupted by noise components, and such kind of speech 'bases' is often overcomplete. Hence there can be many feasible solutions to $Y=Y_{1} S+L+E$. To address this issue, both the most sparse and lowest rank criteria are imposed on $S$. The main consideration is that in the estimated dictionary, speech components have larger or at least comparable magnitudes than noise entries, and the low-rank constraint imposed on $S$ obviously utilizes the similar frequency structures in speech spectral bases. Comparatively, the residual noises in the estimated dictionary can be regarded as sparse components. Thus, we seek a representation $S$ by solving the following optimization problem

$$
\begin{array}{ll}
\min _{S, L} & \|S\|_{*}+\beta\|A\|_{1}+\gamma_{l}\|L\|_{*}+\gamma_{e}\|E\|_{2,1} \\
\text { s.t. } & Y=Y_{1} S+L+E, S=A .
\end{array}
$$

where an auxiliary variable $A$ is introduced to make the objective function separable. $\|E\|_{2,1}=\sum_{i=1}^{M} \sqrt{\sum_{j=1}^{N} E_{i j}^{2}}$ is called the $\ell_{2,1}$ norm. The $\ell_{2,1}$ norm encourages the columns of $E$ to be zero, which assumes that the outlying entries are "sample-specific".

\section{B. Algorithm}

For each row of spectrogram matrix, the probability density function (PDF) is given by

$$
p(y \mid \lambda)=\prod_{j=1}^{M} p\left(y_{j} \mid \lambda\right)
$$

where $p\left(y_{j} \mid \lambda\right)=\sum_{z_{n}} p\left(y_{j} \mid z_{n}, \lambda\right) p\left(z_{n}\right)$. The parameter set $\lambda$ is estimated by maximizing the above PDF function. The following are the typical EM re-estimation formulas

$$
\begin{gathered}
\widehat{w}_{0, n}=\frac{1}{M} \sum_{j=1}^{M} p\left(z_{n} \mid y_{j}, \lambda_{0}^{\prime}\right) \\
\widehat{\mu}_{0, n}=\frac{\sum_{j=1}^{M} y_{j} p\left(z_{n} \mid y_{j}, \lambda_{0}^{\prime}\right)}{M \widehat{w}_{0, n}} \\
\widehat{\sigma}_{0, n}^{2}=\frac{\sum_{j=1}^{M}\left(y_{j}-\widehat{\mu}_{0, n}\right)^{2} p\left(z_{n} \mid y_{j}, \lambda_{0}^{\prime}\right)}{M \widehat{w}_{0, n}}
\end{gathered}
$$

where $p\left(z_{n} \mid y_{j}, \lambda_{0}^{\prime}\right)$ is obtained through (3)-(6) with the old parameter set $\lambda_{0}^{\prime} \cdot \widehat{\lambda}_{0} \sim\left\{\widehat{w}_{0, n}, \widehat{\mu}_{0, n}, \widehat{\sigma}_{0, n}^{2}\right\}$ denotes the new parameter set re-estimated from $\lambda_{0}^{\prime}$. In the next iteration, $\lambda_{0}^{\prime}$ is replaced by $\widehat{\lambda}_{0}$. The initial parameters are obtained by $\mathrm{K}$ means, and the speech model parameter $\nu$ is fixed if the mean values of speech and noise components are too close. This iteration continues until EM algorithm converges. Based on the obtained local SPP, an online updated schematic is proposed [17] as described in Algorithm 1.

Comparing with conventional Gaussian speech model, the generalized Gamma distribution is more restrictive on noise basis spectra and can accurately pick up the speech components [5]. In addition, the online update scheme allows the parameter set to be highly descriptive on local distribution. To solve (7), a recently developed method called the linearized alternating direction method with adaptive penalty (LADMAP) has been applied to obtain the optimization result [18]. The augmented Lagrangian function of (7) is

$$
\begin{aligned}
& \mathcal{L}\left(S, A, L, E, \rho, \Delta_{1}, \Delta_{2}\right) \\
& =\|S\|_{*}+\beta\|A\|_{1}+\gamma_{l}\|L\|_{*}+\gamma_{e}\|E\|_{2,1} \\
& +\frac{\rho}{2}\left\|Y-Y_{1} S-L-E+\frac{\Delta_{1}}{\rho}\right\|_{F}^{2}+\frac{\rho}{2}\left\|S-A+\frac{\Delta_{2}}{\rho}\right\|_{F}^{2}
\end{aligned}
$$

where $\Delta_{1}$ and $\Delta_{2}$ are Lagrangian multipliers. With some algebra, the updating schemes are outlined in Algorithm 2. $\Theta$, $\mathcal{S R}$, and $\Omega$ are the singular value thresholding, shrinkage, and the $\ell_{2,1}$ minimization operator, respectively, and $\eta=\left\|Y_{1}\right\|_{2}^{2}$ [18]. 


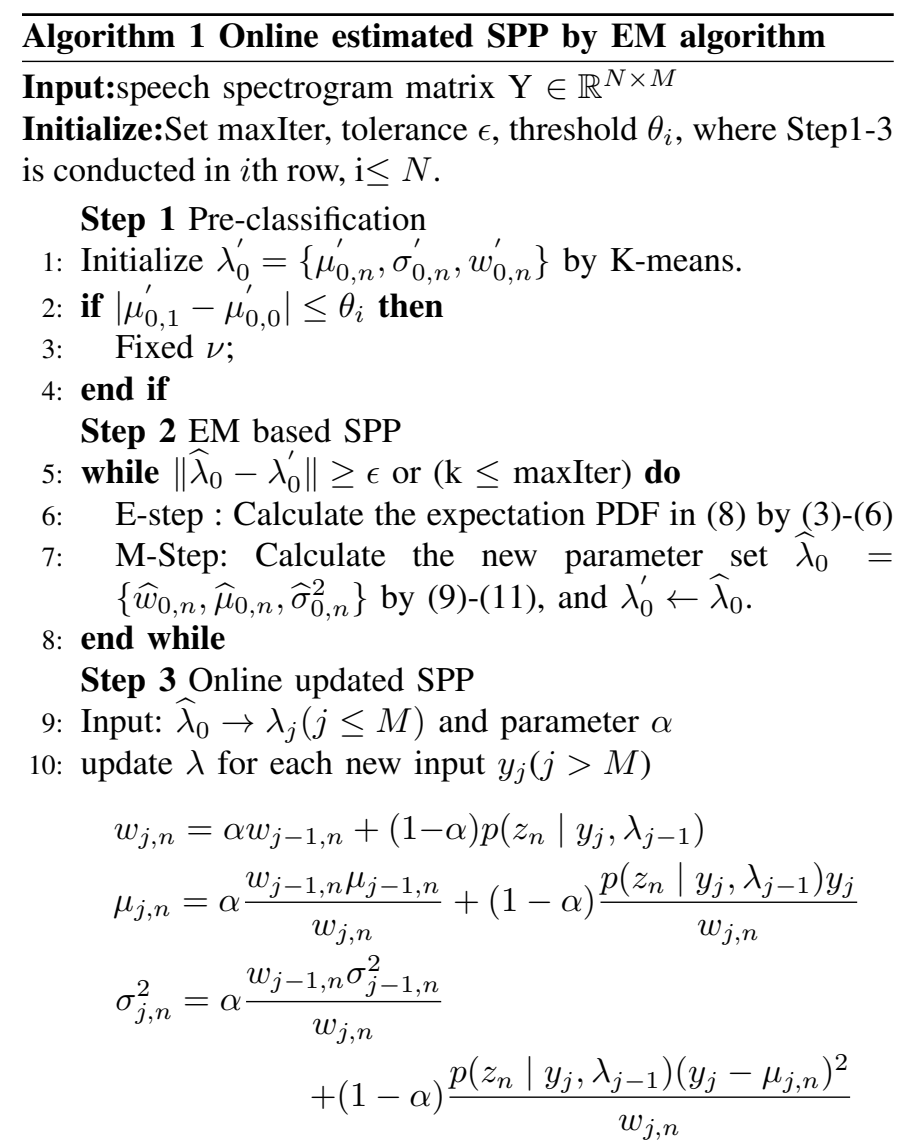

Output:SPP matrix $\mathcal{P}_{i j}=p\left\{z_{1} \mid y_{i j}, \lambda\right\}$

\section{EXPERIMENTAL EVALUATION}

The noisy speech signals were synthesized by adding speech samples to different types of noises at various input SNRs(i.e., $-10,-5,0,5$, and $10 \mathrm{~dB}$ ). Thirty speech samples were selected from NOIZEUS database, and 30 were randomly selected from IEEE wide band speech dataset [9]. Nine different noise samples were used, including six noises (i.e., car, babble, airport, exhibition, restaurant, and train) from AURORA database, two simulated noises (i.e., Gaussian and pink noise), and one jackhammer noise sample from [9]. All signals were resampled to $8 \mathrm{kHz}$ sampling rate, and the spectrograms were calculated with a window length of $32 \mathrm{~ms}$, and a hop of $10 \mathrm{~ms}$. The performance of the proposed LDLSD algorithm was evaluated by comparing with four other algorithms, including one conventional subspace approach (i.e., KLT [7]), and three stateof-the-arts (i.e.,MMSE-SPP [5], NMF-RPCA [2], and RPCA [8]). In NMF-RPCA algorithm, the speech dictionary $Y_{1}$ was learned from the spectrograms of all 60 speech utterances used in this study. Sparse NMF with a generalized KL-divergence [2] was used to obtain the dictionary, which consisted of 300 bases. In other words, 5 basis vectors were extracted for each speech utterance.

An intuitive comparison of the improved speech spectrogram by the proposed LDLSD and two RPCA based algorithms has been shown in Fig.1. For NMF-RPCA, the artificial frequency components can be found around 2.7 second (as circled in Fig.1c). It indicates that the global dictionary

\section{Algorithm 2 Proposed model to solve problem (7)}

Input:Speech spectrum matrix $Y \in \mathbb{R}^{N \times M}$, estimated dictionary $Y_{1}$, parameters $\gamma_{l}>0$ and $\gamma_{e}>0, \rho_{0}>0$, and $\mu>1$.

Initialize:Set maxIter, and tolerance $\epsilon$ False. Initialize $S_{0}, A_{0}$, $L_{0}, E_{0}$ and $\Delta_{0}$ to zero.

$$
\begin{aligned}
& \text { 1: while }\left\|Y-Y_{1} S_{k}-L_{k}-E_{k}\right\|_{F} /\|Y\|_{F} \geq \epsilon \text { or } k \leq \text { maxiter) } \\
& \text { do } \\
& \text { 2: } \quad \text { Update } S_{k+1}, A_{k+1}, L_{k+1}, E_{k+1} \text { : } \\
& S_{k+1}=\Theta_{\frac{1}{\eta \rho_{k}}}\left(\left[Y_{1}^{T}\left(Y-Y_{1} S_{k}-L_{k}-E_{k}+\frac{\Delta_{1, k}}{\rho_{k}}\right)-\right.\right. \\
& \left.\left.\left(S_{k}-A_{k}+\frac{\Delta_{2, k}}{\rho_{k}}\right)\right] / \eta+S_{k}\right) \\
& A_{k+1}=\mathcal{S} \mathcal{R}_{\beta \rho_{k}^{-1}}\left(S_{k+1}+\frac{\Delta_{2, k}}{\rho_{k}}\right) \\
& L_{k+1}=\Theta_{\frac{\gamma_{l}}{\rho_{k}}}\left(Y-Y_{1} C_{k+1}-E_{k}+\frac{\Delta_{1, k}}{\rho_{k}}\right) \\
& E_{k+1}=\Omega_{\frac{\gamma_{e}}{\rho_{k}}}\left(Y-Y_{1} S_{k+1}-L_{k+1}+\frac{\Delta_{1, k}}{\rho_{k}}\right)
\end{aligned}
$$

3: Update the Lagrangian multipliers:

$$
\begin{aligned}
\Delta_{1, k+1} & =\Delta_{1, k+1}+\rho_{k}\left(Y-Y_{1} S_{k+1}-L_{k+1}-E_{k+1}\right) \\
\Delta_{2, k+1} & =\Delta_{2, k+1}+\rho_{k}\left(S_{k+1}-A_{k+1}\right) \\
\rho_{k+1} & =\mu \rho_{k} .
\end{aligned}
$$

\section{4: end while}

Output:Optimal active coefficient matrix $S_{*}=S_{k}$

bases may lead to an overfitting situation. In addition, the overlap of speech and noise basis convex hull can cause speech-similar-structure noise components(as circled around 1 second in Fig.1c). For RPCA, low rank speech spectrum ingredients are very likely to be wrongly decomposed into noise subspace (as circled at the frequency band $0.5-1 \mathrm{kHz}$ in Fig.1f). Comparatively, the proposed LSLSD demonstrates better decomposition results shown in Fig.1g and 1h. The majority of noise components are correctly decomposed into $L$. Moreover, the speech matrix $Y_{1} S$ in Fig. $1 \mathrm{~g}$ includes most low frequency components, and has least signal distortions than the speech matrix obtained by NMF-RPCA and RPCA algorithms in Fig.1c and 1e.

Two metrics, signal-to-distortion ratio (SDR) calculated by BSS_EVAL package and perceptual evaluation of speech quality (PESQ), are used to evaluate speech enhancement algorithms. Figure 2 a shows that the averaged SDRs of the enhanced speeches by applying five different algorithms. The proposed LDLSD algorithm demonstrates the highest SDRs at all SNRs $(-10,-5,0,5$, and $10 \mathrm{~dB})$. It indicates that the proposed algorithm can more effectively separate speech components from background noises. In addition, compared with NMF-RPCA, the LDLSD has a comparable averaged SDR at SNR $=-10 \mathrm{~dB}$, but demonstrates significantly higher averaged SDRs than the NMF-RPCA at other SNRs (i.e., -5, 0,5 , and $10 \mathrm{~dB}$ ).

The PESQ scores of enhanced speeches by five algorithms are shown in Fig.2b. The LDLSD algorithm shows signifi- 


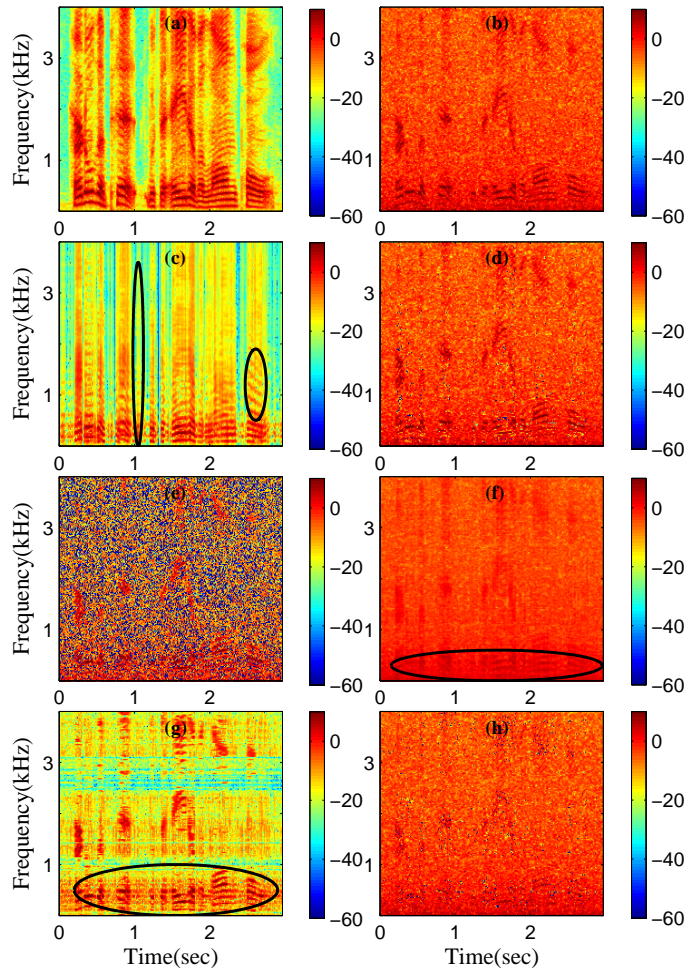

Fig. 1: The spectrograms of clean speech (a), noisy speech with a pink noise $(\mathrm{SNR}=0 \mathrm{~dB})(\mathrm{b})$, speech matrixes obtained by NMF-RPCA (c), RPCA (e), and the proposed LDLSD (g) algorithms, and noise matrixes obtained by NMF-RPCA (d), RPCA(f), and LDLSD (h) algorithms.
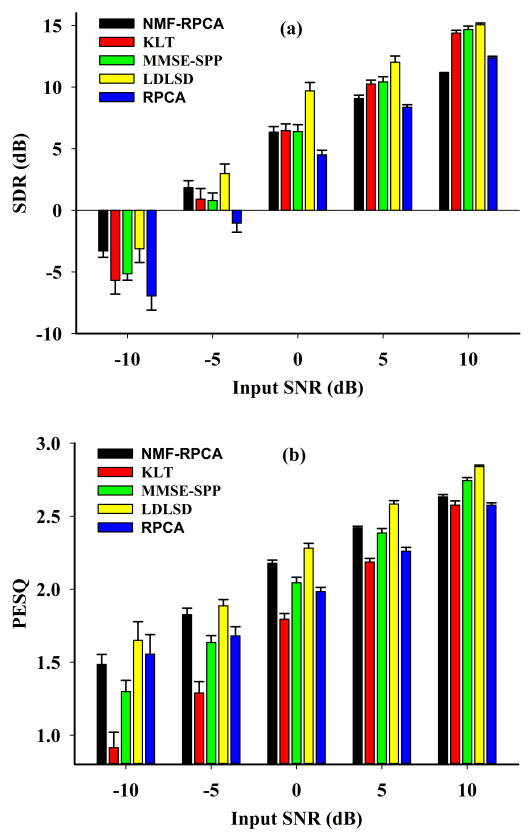

Fig. 2: Averaged SDR (a) and PESQ (b) for enhanced speech by applying five algorithms, including NMF-RPCA, KLT, MMSE-SPP, the proposed LDLSD, and RPCA at various SNRs $(-10 \mathrm{~dB}<$ SNRs $<10 \mathrm{~dB})$, and averaged across eight different types of noise.

cantly higher PESQ improvements than other four algorithms, averagely 0.2 higher than NMF-RPCA, 0.3 higher than RPCA, and 0.4 higher than KLT. Especially at low SNRs (-10 and -5
$\mathrm{dB})$, the low rank and sparse criteria imposed on the activation matrix $S$ help achieve a better performance than NMF-RPCA algorithm.

In addition, the jackhammer noise, as a highly transient noise, is applied to evaluate the performance of the proposed algorithm. Figure 3 shows the SDRs and PESQ of the enhanced speeches from a jackhammer noise background at various SNRs by five algorithms. The LDLSD demonstrates an obvious advantage over other four algorithms on both two metrics. In magnitude spectra space, the transient noises (e.g., jackhammer noises) and global speech dictionary may be partially overlapped, for example the impulsive components are quite similar to the speech fricatives. This can cause an ambiguity in speech and noise separation in NMF-RPCA. Comparatively, the proposed LDLSD algorithm has two obvious merits: 1) the local estimated exemplar can help to exclude most of the transient features; 2) the low rank constraint imposed on the activation matrix $S$ can also reduce the impact of transient noise residuals in the online estimated dictionary $Y_{1}$.
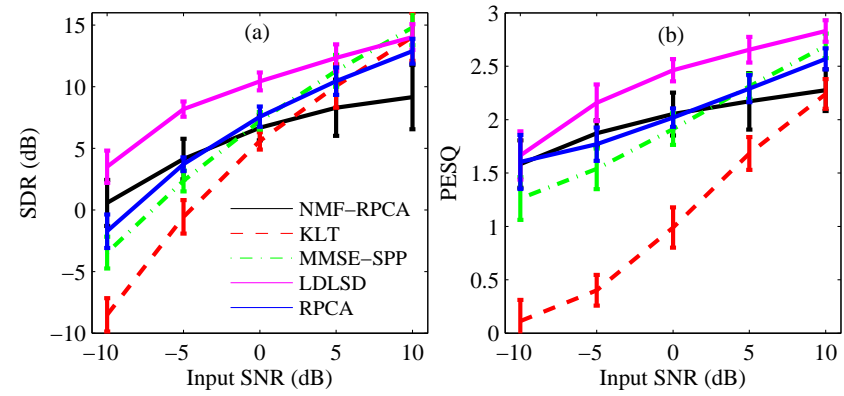

Fig. 3: Averaged SDR (a) and PESQ (b) for enhanced speech corrupted by transient jackhammer noises, by applying five algorithms.

\section{CONCLUSION}

In this letter, we investigate how the SPP based local speech dictionary can be employed in the subspace framework to obtain the low rank and sparse components of speech spectrogram for noisy speech enhancement. A local dictionary based low rank and sparsity decomposition has been proposed to separate the noise and speech components. An online updated EM algorithm is introduced to obtain SPP matrix according to the input noisy speech matrix. By multiplying this SPP matrix element-wise, the broad bases in the speech subspace can be reduced, which consequently improves the accuracy of local speech dictionary. Moreover, the online estimated dictionary is sufficient enough in basis subspace to avoid speech distortion. Specifically, the most sparsity and lowest rank criteria are both imposed to the activation matrix to achieve a noise-resistant decomposition. The results show that LDLSD algorithm obtains significant improvements at various SNRs w.r.t SDR and PESQ, compared with four algorithms, including KLT, MMSE-SPP, NMF-RPCAR and PCA. The future work of this study includes investigation on noise constraints, such as noise variance and noise modeling.

\section{REFERENCES}

[1] Aïcha Bouzid, Noureddine Ellouze, et al. Speech enhancement based on wavelet packet of an improved principal component analysis. Computer Speech \& Language, 35:58-72, 2016. 
[2] Zhuo Chen and Daniel PW Ellis. Speech enhancement by sparse, low-rank, and dictionary spectrogram decomposition. In Applications of Signal Processing to Audio and Acoustics (WASPAA), 2013 IEEE Workshop on, pages 1-4. IEEE, 2013.

[3] Zhiyao Duan, Gautham J Mysore, and Paris Smaragdis. Speech enhancement by online non-negative spectrogram decomposition in nonstationary noise environments. In INTERSPEECH, pages 595-598, 2012.

[4] Ehsan Elhamifar and Rene Vidal. Sparse subspace clustering: Algorithm, theory, and applications. Pattern Analysis and Machine Intelligence, IEEE Transactions on, 35(11):2765-2781, 2013.

[5] Richard C Hendriks, Timo Gerkmann, and Jesper Jensen. Dft-domain based single-microphone noise reduction for speech enhancement: A survey of the state of the art. Synthesis Lectures on Speech and Audio Processing, 9(1):1-80, 2013.

[6] Richard C Hendriks and Rainer Martin. Map estimators for speech enhancement under normal and rayleigh inverse gaussian distributions. Audio, Speech, and Language Processing, IEEE Transactions on, 15(3):918-927, 2007.

[7] Yi Hu and Philipos C Loizou. A generalized subspace approach for enhancing speech corrupted by colored noise. Speech and Audio Processing, IEEE Transactions on, 11(4):334-341, 2003.

[8] Zhouchen Lin, Minming Chen, and Yi Ma. The augmented lagrange multiplier method for exact recovery of corrupted low-rank matrices. arXiv preprint arXiv:1009.5055, 2010.

[9] Philipos C Loizou. Speech enhancement: theory and practice. CRC press, 2013.

[10] Nasser Mohammadiha and Simon Doclo. Single-channel dynamic exemplar-based speech enhancement. In INTERSPEECH, pages 2690 2694, 2014.

[11] Nasser Mohammadiha, Paris Smaragdis, and Arne Leijon. Supervised and unsupervised speech enhancement using nonnegative matrix factorization. Audio, Speech, and Language Processing, IEEE Transactions on, 21(10):2140-2151, 2013.

[12] Pablo Sprechmann, Alexander Bronstein, and Guillermo Sapiro. Supervised non-euclidean sparse nmf via bilevel optimization with applications to speech enhancement. In Hands-free Speech Communication and Microphone Arrays (HSCMA), 2014 4th Joint Workshop on, pages 11-15. IEEE, 2014.

[13] Pablo Sprechmann, Alexander M Bronstein, and Guillermo Sapiro. Realtime online singing voice separation from monaural recordings using robust low-rank modeling. In ISMIR, pages 67-72, 2012.

[14] Chengli Sun, Qi Zhu, and Minghua Wan. A novel speech enhancement method based on constrained low-rank and sparse matrix decomposition. Speech Communication, 60:44-55, 2014.

[15] Meng Sun, Yinan Li, Jort Gemmeke, et al. Speech enhancement under low snr conditions via noise estimation using sparse and low-rank nmf with kullback-leibler divergence. Speech and Audio Processing, IEEE Transactions on, 23(7):1233-1242, 2015.

[16] Dalei Wu, Wei-Ping Zhu, and MNS Swamy. The theory of compressive sensing matching pursuit considering time-domain noise with application to speech enhancement. Audio, Speech, and Language Processing, IEEE/ACM Transactions on, 22(3):682-696, 2014.

[17] Dongwen Ying, Yonghong Yan, Jianwu Dang, and Frank K Soong. Voice activity detection based on an unsupervised learning framework. Audio, Speech, and Language Processing, IEEE Transactions on, 19(8):26242633, 2011.

[18] Liansheng Zhuang, Haoyuan Gao, Zhouchen Lin, Yi Ma, Xin Zhang, and Nenghai Yu. Non-negative low rank and sparse graph for semisupervised learning. In Computer Vision and Pattern Recognition (CVPR), 2012 IEEE Conference on, pages 2328-2335. IEEE, 2012. 